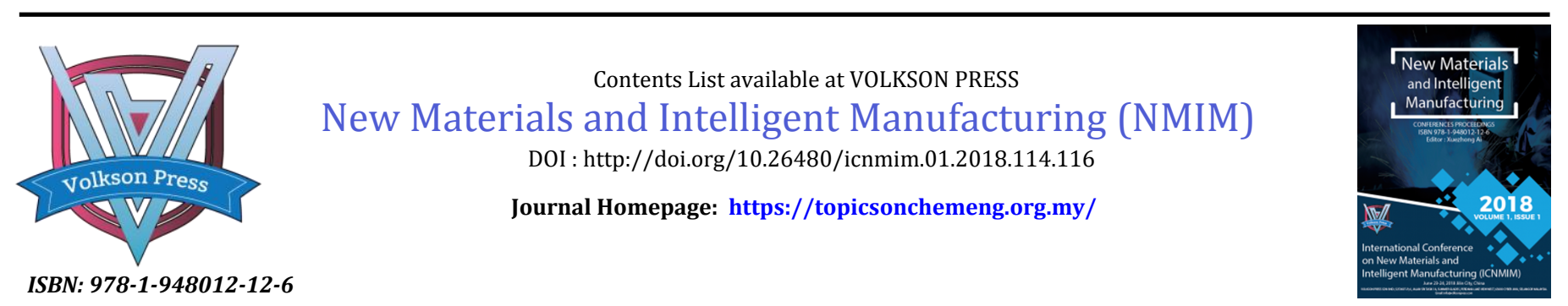

\title{
GREEN SYNTHESIS OF MCM-49 ZEOLITE USING LOW TOXICITY TEMPLATE
}

\author{
Qin Tizhi, Jiang Di, Jiang Wenjing, Li Zhaoxu, Lu Hongyu and Zhang Yu* \\ Jilin Institute of Chemical Technology, Jilin City, China \\ *Corresponding Author Email: Zhang Yu: zhang99yu@hotmail.com
}

This is an open access article distributed under the Creative Commons Attribution License, which permits unrestricted use, distribution, and reproduction in any medium, provided the original work is properly cited.

\section{ARTICLE DETAILS}

\section{Article History:}

Received 26 June 2018 Accepted 2 July 2018 Available online 1 August 2018

\section{ABSTRACT}

MCM-49 zeolites have been synthesized by dynamic hydrothermal method with piperazine (PIP) as low toxicity template to replace traditional cancerogenic hexamethyleneimine (HMI). The structure and physical-chemical properties of synthesized samples were studied by means of various characterization techniques, such as XRD, SEM, $\mathrm{N}_{2}$ adsorption-desorption, and $\mathrm{NH}_{3}$-TPD. The effects of PIP to HMI ratio in composite template were investigated. Results show that high crystalline and high purified MCM-49 zeolites could be synthesized using PIP as template to completely or partially replace HMI.

\section{KEYWORDS}

MCM-49 zeolite, template, piperazine, hexamethyleneimine.

\section{INTRODUCTION}

MCM-49 zeolite is a branch of MWW topological structure zeolites with a layered morphology, Which has three kinds of complex pore systems: (1)12 membered ring cups $(0.71 \mathrm{~nm} \times 0.71 \mathrm{~nm} \times 0.91 \mathrm{~nm})$ located on the external surface of the lamellar zeolite; (2) Interlayer 12 MR supercages $(0.71 \mathrm{~nm} \times 0.71 \mathrm{~nm} \times 1.82 \mathrm{~nm})$ opening through $10 \mathrm{MR}$ windows; (3) and interlayer two-dimensional sinusoidal $10 \mathrm{MR}$ pores $(0.41 \mathrm{~nm} \times 0.54 \mathrm{~nm})$ [1-7]. Especially, numerous $12 \mathrm{MR}$ cups on the external surface of layered structure endow this materials with unique properties different from bulk molecular sieves [8]. Owing to its unusual porous structure, high micro porosity, high hydrothermal stability and mild acid property, MCM-49 has demonstrated good catalytic performance on many reactions, such as alkylation, aromatization, disproportionation of toluene, catalytic cracking, etc [9-12].

MCM-49 was dynamically synthesized by the hydrothermal process. The traditional organic template used in the synthesis of MCM-49 zeolite is HMI, which is highly poisonous, cancerogenic and expensive [13]. Therefore, it is necessary to explore a kind of cheap and low toxicity template to replace HMI completely or partially [14].

In this paper, piperazine (PIP) and PIP/HMI mixtures were used respectively as single or mixed low toxicity template to prepare MCM-49. All samples were characterized by means of XRD, SEM, BET, $\mathrm{N}_{2}$ adsorptiondesorption, and $\mathrm{NH}_{3}$-TPD in order to clarify the effect of PIP to HMI ratio on the structure, morphology and acid properties of MCM-49.

\section{EXPERIMENTAL}

\subsection{Materials Preparation}

MCM-49 zeolites was synthesized from a gel of the following composition: $n_{\mathrm{SiO} 2}: n_{\mathrm{Al} 203}: n_{\mathrm{NaOH}}: n_{\text {(HMI+PIP) }}: n_{\mathrm{H} 2 \mathrm{O}}=1: 0.05: 0.12: 0.35: 18.7 . \mathrm{NaAlO}_{2}$ and silica gel (25 wt\%) were used as the source of $\mathrm{SiO}_{2}$ and $\mathrm{Al}_{2} \mathrm{O}_{3}$, respectively. The mixtures of HMI and PIP were used as the structure directing agent (SDA). under violent stirring. HMI and PIP composite template was added dropwise to the gel. The synthesis gel was stirred with a magnetic stir bar at room temperature for $2 \mathrm{~h}$. The product was loaded into teflon lined $150 \mathrm{ml}$ stainless-steel autoclave and placed in a convection oven at $448 \mathrm{~K}$ for $72 \mathrm{~h}$. Autoclaves were tumbled at $12 \mathrm{r} / \mathrm{min}$ to improve the mixing of the synthesis gel. After $72 \mathrm{~h}$, the autoclaves were cooled to room temperature and the gel was filtered and washed with a large quantity of deionized water. The as-made zeolite was dried at $373 \mathrm{~K}$ overnight, and calcined at $813 \mathrm{~K}$ for $6 \mathrm{~h}$.

\subsection{Catalystic Characterization}

Powder X-ray diffraction (XRD) patterns were collected to estimate the structural phase and crystallinity of the synthesized materials on a Bruker D/Max-2500 diffractometer using CuK $\alpha$ radiation, operating at $40 \mathrm{kV}$ and $40 \mathrm{~mA}$. Scanning electron microscopic (SEM) images were recorded on a Hitachi X-650 microscope. Specific surface and pore size were recored on 3H-2000PM1 Specific surface \& pore size analysis instrument. Temperature-programmed desorption of ammonia $\left(\mathrm{NH}_{3}-\mathrm{TPD}\right)$ was carried out on a Finesorb 3010D chemisorption apparatus.

\section{RESULT AND DISCUSSION}

\subsection{XRD}

Figure 1 shows the X-ray patterns of MCM- 49 with different PIP content in composite tempalte $(0 \%, 40 \%, 70 \%, 100 \%)$. The XRD patterns of all samples are in good agreement with those previously reported [15]. The peak widths between 7 10 and 20 30 degree indicate that MCM-49 is composed of thin layers extending in $a$ and $b$ direction [9]. XRD analysis indicates that MCM- 49 could be successfully obtained by completely or partially replace of HMI with PIP as low toxicity template. The ratio of PIP to HMI doesn't affect the crystallinity of MCM-49 zeolite. 


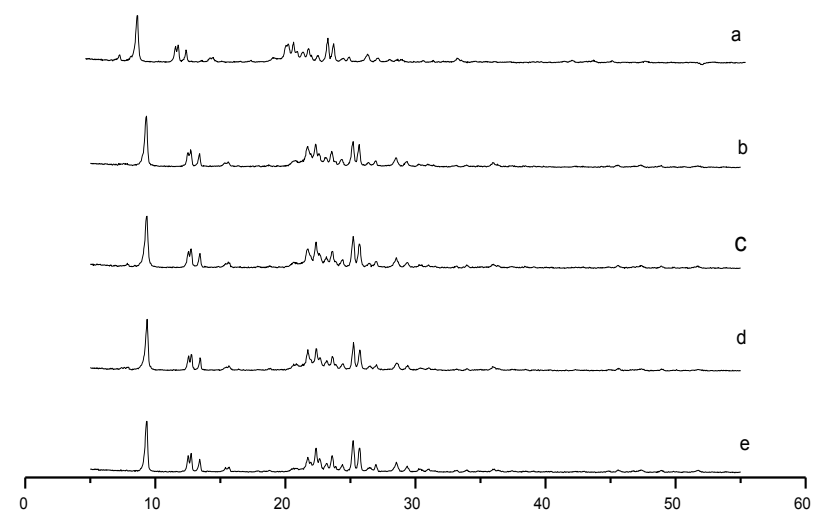

Figure 1: XRD pattern of (a)MCM-49(0\%PIP); (b)MCM-49(40\%PIP); (c)MCM-49(50\%PIP); (d)MCM-49(70\%PIP); (e)MCM-49(100\%PIP).

\subsection{SEM}

The scanning electron micrographs of MCM-49 (0\%PIP), MCM-49 (40\%PIP), MCM-49 (70\%PIP) and MCM-49 (100\%PIP) are shown in Figure 2. The particles of all samples look like aggregates of sheet-like structures which do not have a well-defined morphology at the micron scale. There is no obvious difference in the surface morphology between MCM-49 (0\%PIP) and MCM-49 (100\%PIP) can be discerned according to these images.

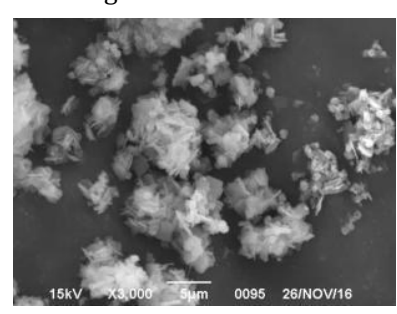

(a)

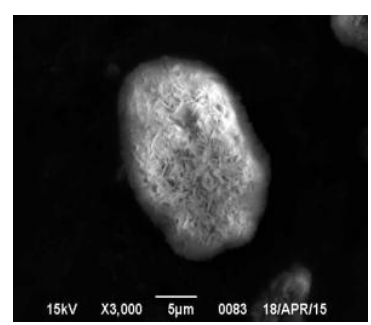

(c)

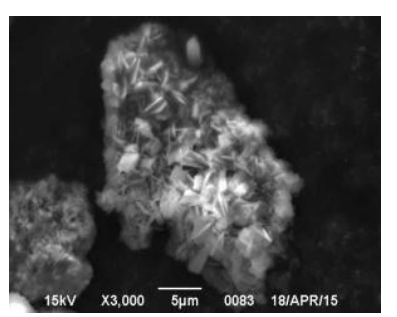

(b)

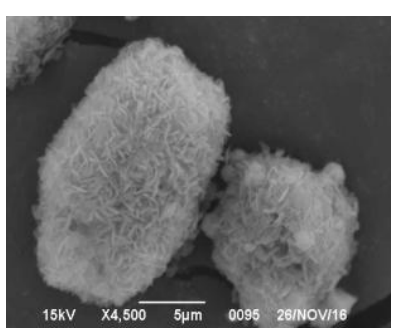

(d)

\subsection{BET}

The nitrogen adsorption-desorption isotherms of samples are presented in Figure 3, which indicates that both MCM-49(0\%PIP) and MCM$49(40 \%$ PIP) are microporous materials. The inflection point appears at 0.1 relative pressure. The specific surface area and pore volume of zeolites are shown in Table 1. The BET surface areas are quite different for MCM49 (0\%PIP) and MCM-49 (40\%PIP) at 439 and $424 \mathrm{~m}^{2} / \mathrm{g}$ respectively. Compared to MCM-49 (0\%PIP), the external surface area of MCM-49 ( $40 \% \mathrm{PIP})$ is similar, which indicates that after applying PIP , there is no obvious difference in the external surface area and the t-plot micropo volume.

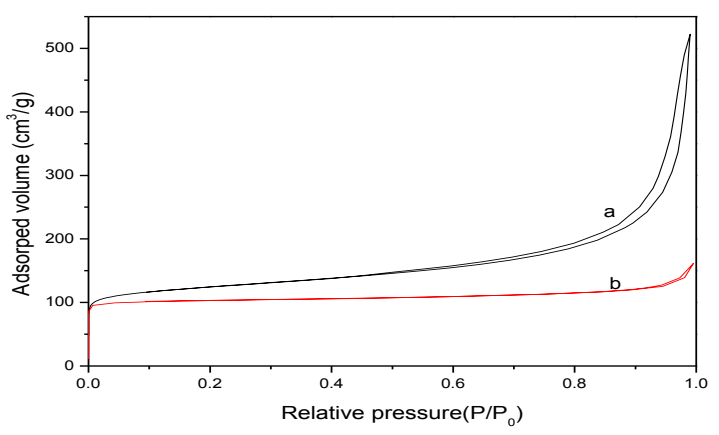

Figure 3: Nitrogen adsorption-desorption isotherm of (a) MCM-49 (0\%PIP) and (b) MCM-49(40\%PIP).

Figure 2: Scanning electron micrographs of (a) MCM-49(0\%PIP); (b) MCM-49(40\%PIP); (c) MCM-49(70\%PIP); (d) MCM-49(100\%PIP).

Table 1: Structural parameter of of MCM-49 zeolites

\begin{tabular}{|llccc|}
\hline Sample & $\begin{array}{c}\mathrm{S}_{\text {BET }} \\
{\left[\mathrm{m}^{2} \cdot \mathrm{g}^{-1}\right]}\end{array}$ & $\begin{array}{l}\mathrm{S}_{\mathrm{t} \text {-plotexternal }} \\
{\left[\mathrm{m}^{2} \cdot \mathrm{g}^{-1}\right]}\end{array}$ & $\begin{array}{l}\mathrm{V}_{\text {Total }} \\
{\left[\mathrm{cm}^{3} \cdot \mathrm{g}^{-1}\right]}\end{array}$ & $\begin{array}{c}\mathrm{V}_{\text {t-plot micropo }} \\
{\left[\mathrm{cm}^{3} \cdot \mathrm{g}^{-1}\right]}\end{array}$ \\
\hline 0\%PIP & 439 & 83 & 0.58 & 0.19 \\
$40 \%$ PIP & 424 & 97 & 0.52 & 0.15 \\
\hline
\end{tabular}

\section{$3.4 \quad \mathrm{NH}_{3}-\mathrm{TPD}$}

The acid properties of MCM-49 (0\%PIP) and MCM-49 (40\%PIP) were characterized by temperature-programmed desorption (TPD) of ammonia (Figure 4). Both $\mathrm{NH}_{3}$-TPD profiles show two peaks at around $220 \sim 250^{\circ} \mathrm{C}$ and $350 \sim 390^{\circ} \mathrm{C}$, which can be attributed to the presence of weak acidic sites and relatively strong acid sites (mainly Brønsted acidity), respectively. It can be seen from Figure 4 that the acid strength distribution of these two samples is similar. However, MCM-49(40\%, PIP) shows a slightly less total acid amounts than MCM-49(0\%, PIP).

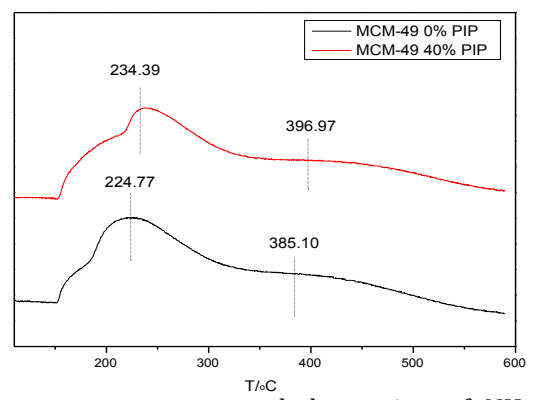

Figure 4: Temperature-programmed desorption of $\mathrm{NH}_{3}$ for MCM-49 (0\%PIP) and HMCM-49 (40\%PIP) 


\section{CONCLUSIONS}

MCM-49 molecular sieves with high crystallinity and high purity could be synthesized by dynamic hydrothermal method using PIP as low toxicity template to completely or partially replace traditional cancerogenic HMI. There is no obvious difference in the external surface areas and micropo volume by introduce PIP. MCM-49 prepared with PIP as template shows a similar acid strength distribution and a slightly less total acid amounts. This indicates that PIP is a potential environment-friendly template, which could be applied in the industrial production of MCM- 49 .

\section{ACKNOWLEDGE}

We are grateful to Technological Innovation Development Project of Jilin city (20161205) and Technological Development Project of Jilin Institute of Chemical Technology (2015018) for financial support.

\section{REFERENCES}

[1] Bennett, J.M., Chang, C.D., Lawton, S.L. 1993. Synthetic porous crystalline MCM, 49, its synthesis and use, US 5236575.

[2] Roth, W.J., Dorset, D.L. 2011. Expanded view of zeolite structures and their variability based on layered nature of 3-D Framework. Microporous Mesoporous Mater, 142, 32-36.

[3] Gao, N.N., Xie, S.J., Liu, S.L. 2014. Catalytic Degradation of LDPE and PP over MCM-49 Based Micro-Mesoporous Composites. Catalysis Letters, 144, 1296-1304

[4] Ningning, G., Sujuan, X., Shenglin, L., Jie, A., Xiangxue, Z., Linyan, H., Huijuan, W., Xiujie, L., Longya, X. 2014. Catalysis Letters, 144 (7), 1296-1304.

[5] Bellussi, G., Carati, A., Rizzo, C., Millini, R. 2013. New trends in the synthesis of crystalline microporous materials. Catalysis Science and Technology, 3 (4), 833- 857.

[6] Frillette, V.J., Haag, W.O., Lago, R.M. 1981. Catalysis by crystalline aluminosilicates: Characterization of intermediate pore-size zeolites by the "Constraint Index". Journal of Catalysis, 67 (1), 218-222.

[7] Weisz, P.B., Frilette, V.J. 1960. Intracrystalline And MolecularShape-Selective Catalysis by Zeolite Salts. The journal of physical chemistry, 64 (3), 382-382.

[8] Liu, K., Xie, S., Xu, G., Li, Y., Liu, S., Xu, L. 2010. Appl Catal A 383, 102.

[9] Corma, A., Corell, C., Flopis, F., Martı́nez, A., Pe'rez- Pariente, J. 1994. Appl.Catal A 115, 121.

[10] Corma, A., Martı́nez-Triguero, J. 1997. J. Catal. Applied Catalysis A: General,165,102.

[11]Wu, P., Komatsu, T., Yashima, T. 1998. Micropor. Mesopor. Mater., 22, 343.
[12] Abril, R.P.L., Bewes, E., Green, G.J., Marler, D.O., Sihabi, D.S., Sacha, R.F. 1992. US Patent 5085762.

[13] Bennete, Michael, Chang. 1993. Synthetic porous crystalline MCM-49, its synthesis and use: US, $5236575[\mathrm{P}]$.

[14] Mochida, I., Eguchi, S., Hironaka, M., Nagao, S., Sakanishi, K., Whitehurst, D.D. 1997. Zeolites 18, 142.

[15] Barrer, R.M. 1989. Zeolites: their nucleation and growth, ACS Symp Ser 398 [C], ACS, Washington.

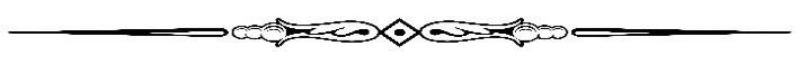

